\title{
The Asymmetry and Modularity of the Hyoid Bone
}

\author{
Asimetría y Modularidad del Hueso Hioides
}

\author{
Petra Urbanová*; Petr Hejna**; Lenka Zátopková** \& Miroslav Safr**
}

URBANOVÁ, P.; HEJNA, P.; ZÁTOPKOVÁ, L. \& SAFR, M. The asymmetry and modularity of the hyoid bone. Int. J. Morphol., 32(1):251-260, 2014.

SUMMARY: Morphological variation is a result of interplay among multiple intervening factors. For hyoid bones, the shape and size differences have been scarcely covered in the literature and in majority limited to studies of sexual dimorphism or age dependency. To our knowledge, the human hyoid bone, in complete opposite to other cranial bones, has not been fully utilized to address development questions in terms of asymmetry or modularity. In the present paper, we used landmark-based methods of geometric morphometrics and multivariate statistical approach to study human hyoid morphology represented by the hyoid body and greater horns in a sample of 211 fused and non-fused bones. Within a sample variation analysis, we showed that the hyoid bone is, by nature, asymmetrical bone which exhibits both directional and fluctuating types of asymmetry and is composed of well-integrated anatomical elements for which the biomechanical load of attached muscles is the most determining factor of variation. Yet, the covariance and evidence of unequal amount of fluctuating asymmetry among modules suggests a certain degree of independence during early stages of development.

KEY WORDS: Hyoid bone; Asymmetry; Modularity; Geometric morphometrics.

\section{INTRODUCTION}

In the literature, the hyoid bone is a rather neglected structure of the human skeleton which has not been given sufficient attention. Functionally, the hyoid bone serves as an attachment of supra- and infra-hyoid muscles taking part in mastication and swallowing. Anatomically, the bone consists of five elements, an unpaired body, and pairs of greater and lesser horns. All elements originate in cartilaginous tissue of the pharyngeal (also known as brachial) arches. By a generally accepted concept of origin, the lesser horns and superior part of the body above the vertical ridge are derived from the second, so-called hyoid arch, while the rest of the body and greater horns differentiate from the third pharyngeal arch (Scheuer \& Black, 2000).

Previous morphological studies of the hyoid bone focused primarily on describing general shape and size variations (Pollanen \& Ubelaker, 1997; Miller et al., 1998). It has been noted that size and shape is modified by functional demands and is affected by the individual's sex (Kindschuh et al., 2010), age (Gupta et al., 2008; Urbanová et al., 2013a), ancestry (Kindschuh et al., 2010; Kindschuh et al., 2012) and to a lesser extent by body size parameters (Urbanová et al., 2013b).
Like most human skeletal structures, the hyoid bone is generally assumed to be bilaterally symmetrical. The leftright symmetry of hyoid bones corresponds to object symmetry, where a single structure is identical according to a given or selected plane, such as mid-sagittal plane. The matching symmetry, in contrast, is referred to in situations where two separate objects exist as mirror images of each other (Klingenber et al., 2002).

The disturbances in symmetry and an occurrence of asymmetry within data might be an indicator of individual or population-related developmental stress, shed light on pathological conditions or indicate a relation between structurally or functionally interacting elements. The evidence of asymmetry in the human hyoid bone has been explored in relation to the individual's sex (Pollanen \& Ubelaker) and body size (Urbanová et al., 2013b). Recently, symmetry/asymmetry issues have been explored owing to the geometric morphometrics (GM). GM has been primarily helpful in standardizing the manner in which the symmetry is investigated and in separating three different sources of asymmetry - directional, fluctuating asymmetry and antisymmetry (Palmer \& Strocker, 1986).

\footnotetext{
* Department of Anthropology, Faculty of Science, Masaryk University, Brno, Czech Republic.

** Institute of Legal Medicine, Medical Faculty of Charles University and University Hospital Hradec Králové, Hradec Králové, Czech Republic.
} 
Klingenberg (2008) showed that within the concept of geometric morphometrics studies of asymmetry can be combined with other morphological issues - modularity and integrity. While modularity refers to the covariance among morphological structures that originates in independent developmental processes, so-called modules, integration, a counterpart to modularity, is a measure of the interconnection among parts in order to function as a whole (Klingenberg, 2008). Modules can be defined with respect to genetic, developmental, functional or evolutionary context. While studying adult structures, for instance, the modularity allows extrapolating morphological data to answer a question on how the traits or parts interacted developmentally. The magnitude of interactions among modules is customarily expressed as a function of their covariance. If the true boundary between modules is weak meaning that two units in question are reasonably independent, then the degree of covariance will be accordingly low. In contrast, two modules linked into a strongly interrelated system will provide a higher value of covariance.

In the present paper we use geometric morphometrics to quantify the observed degree of asymmetry in the sample of fused and non-fused human hyoid bone and to identify those morphological characteristics that are modular and those that are integrated in the system throughout anatomical, functional or developmental interactions.

\section{MATERIAL AND METHOD}

The studied sample was composed of 211 hyoid bones extracted from individuals of Czech origin at medico-legal autopsies. Both fused and non-fused bones were included in the study. The distribution of sex was slightly skewed towards males (117:94). The sample was divided into 4 age groups: less than 30 years, 31 to 50 years, 51 to 70 years and more than 71 years (Table I). Only adults were incorporated in the study. The hyoid morphology was described by a set of 23 landmarks (Fig.1) covering the body and the greater horns (lesser horns were not incorporated). The Cartesian coordinates of all 23 landmarks were recorded by MicroScribe G2LX digitizer with a bone or a non-fused element mounted carefully on a handler. The set of Cartesian coordinates was further standardized by the generalized Procrustes fitting.

A/symmetry in size. The size of the bones was expressed as values of the centroid size. The centroid size is a sideproduct of the generalized Procrustes fitting and is computed as the average distance between landmarks and the center of gravity of a given configuration.
Table I. Age distribution within studied sample.

\begin{tabular}{lcc}
\hline \multicolumn{1}{c}{ Age category (years) } & $\mathbf{n}$ & $\boldsymbol{\%}$ \\
\hline$<30$ & 7 & 3.32 \\
$31-50$ & 29 & 13.74 \\
$51-70$ & 99 & 46.92 \\
$>70$ & 76 & 36.02 \\
Total & 211 & 100.000 \\
\hline
\end{tabular}

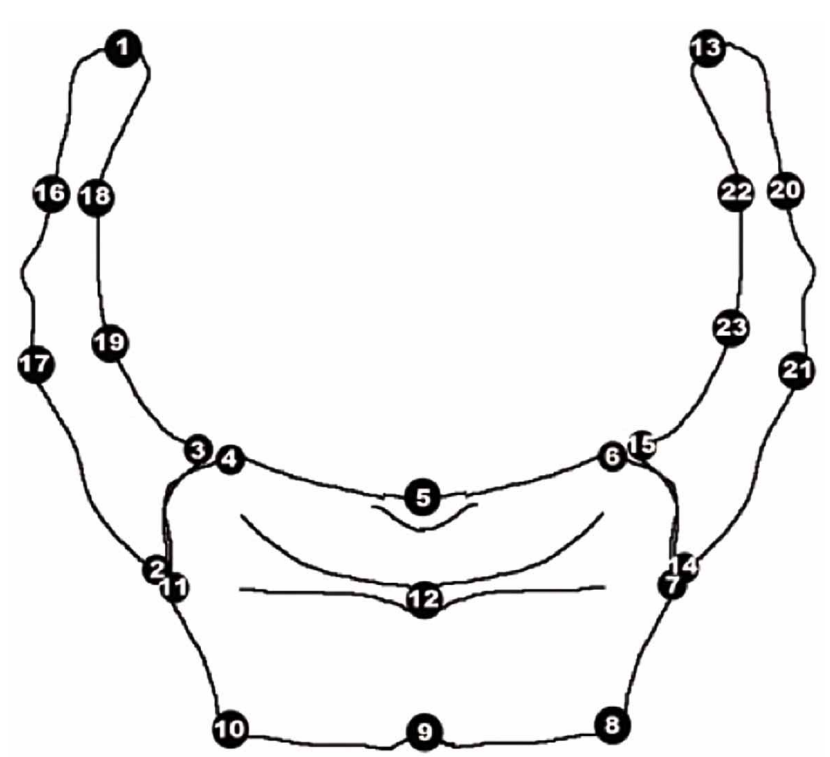

Fig. 1. Scheme illustrating a set of recorded landmarks: $1-$ posterior end of the right greater horn, 2 - medial inferior end of the right greater horn, 3 - medial superior end of the right greater horn, 4 right lateral superior corner of the body, 5 - superior middle point of the body, 6 - left lateral superior corner of the body, 7 - left lateral inferior corner of the body, 8 - the most lateral and inferior left point of the inferior margin, 9 - inferior middle point of the body, 10 - the most lateral and inferior right point of the inferior margin, 11 - right lateral inferior corner of the body, 12 - the most anterior point of the horizontal ridge located in mid-sagittal plane, 13 - posterior end of the left greater horn, 14 - medial inferior end of the left greater horn, 15 - medial superior end of the left greater horn, 16 lateral (inferior) margin of the right greater horn in the posterior third, 17 - lateral (inferior) margin of the right greater horn in the anterior third, 18 - medial (superior) margin of the right greater horn in the posterior third, 19 - medial (superior) margin of the right greater horn in the anterior third, 20 - lateral (inferior) margin of the left greater horn in posterior third, 17 - lateral (inferior) margin of the left greater horn in anterior third, 18 - medial (superior) margin of the left greater horn in the posterior third, 19 - medial (superior) margin of left greater horn in the anterior third.

In order to test object a/symmetry in size, the original configuration of 23 landmarks was subdivided into 2 configurations of 13 landmarks each containing a set of points from only right or left body side (10 for each side) 
plus 3 mid-sagittal landmarks. For each configuration values of the centroid size was computed and tested against each other by t-test (directional asymmetry) and paired t-test (fluctuating asymmetry). Furthermore, the right-to-left scores (computed as CSR-CSL, where CSR is a value of the centroid size of the right-sided configuration and CSL is an equivalent for the left-sided configuration) were tested in respect to individual's sex and age categories by t-test and ANOVA. If test assumptions were not met, non-parametric alternatives were processed (Mann-Whitney U test, Kruskal-Wallis test).

Matching a/symmetry was explored on a set of greater horns described by a configuration of 7 landmarks. Two separated analyses were carried out, one on non-fused bones only, and the second where the dataset was pooled with subdivisions of the total configuration of the fused hyoids. The directional and fluctuating asymmetry was tested accordingly to the object asymmetry, i.e., t-test, paired t-test, ANOVA or non-parametric alternatives.

A/symmetry in shape. In order to explore a/symmetry in shape of fused bones the variance of the standardized Cartesian coordinates was sub-divided into two components, symmetrical and asymmetrical. The symmetrical component included displacement of unpaired landmarks along the midsagittal axis and averaged right and left paired landmarks moving in any direction. It is, in fact, the correction for studying bilateral data if the symmetry/asymmetry is not a primary focus. The asymmetrical component is qualified as a difference between the original and the ideally symmetrical configuration included into the symmetrical component. A displacement of unpaired landmarks is allowed in the direction perpendicular to the mid-sagittal axis while paired landmarks move freely. In addition to the total configuration of points, a configuration including landmarks of hyoid body was processed.

Object a/symmetry in the shape of fused hyoid bones was tested by the Procrustes ANOVA with the specimen's ID and side defined as factors. While "ID" effect counts individual variation throughout the symmetrical component, "side" effect reflects the directional asymmetry via the asymmetrical component. A combination of both effects sums up the fluctuating asymmetry. Fluctuating asymmetry was expressed by the Procrustes fluctuating asymmetry scores and tested against sex and age factors by the MannWhitney $U$ test and one-way ANOVA respectively. In addition to the subset of fused bones, a configuration of body landmarks (pooled fused and non-fused bones) was processed in the same manner.

Matching symmetry was examined on a configuration of 7 landmarks for the greater horns of each body side by the Procrustes ANOVA. Prior to the analysis left bones were left-to-right reflected and the Procrustes fit was performed on a pooled configuration incorporating both right and left greater horns. As both configurations become detached, their spatial arrangement in relations to the body and each other is lost in the process. As a consequence, the approach tests for asymmetry of the shape per se regardless of their in vivo orientation and spatial arrangement. Like object symmetry, this approach enables distinguishing between directional and fluctuating asymmetry. The mean of the asymmetrical component was subsequently ascribed to directional asymmetry, whereas fluctuating asymmetry was expressed by the Procrustes fluctuating asymmetry scores and variation in regards to sex, age and fusion was tested by the MannWhitney U test and one-way ANOVA test respectively. Both fused and non-fused bones were processed.

Modularity. To localize boundaries in the hyoid bones, the total configuration of 23 landmarks was divided into a variety of subsets and treated as separate modules. Only fused bones were processed. Three main hypotheses were tested: covariance of true anatomical elements (i.e. greater horns and body), covariance of compartments of identical embryonic origin (greater horns, upper and lower parts of the body) and covariance of superior and inferior halves (Table III). The extent of covariance was expressed in terms of the RV coefficient. The assumption was that the covariance between true modules should be lower than between other theoretically possible alternative partitions of the same number of landmarks. Alternatively, if the hyoid bone forms a single homogeneous module, then all subsets throughout the bone should co-vary with one another. The probability of the RV coefficient was acquired by permutation test with an arbitrary number of 10000 repeats. Only landmarks forming a continuous shape were allowed to form random configurations. Both symmetrical and asymmetrical components were processed.

The Procrustes fit and most of the statistics were executed by using MorphoJ software (Klingenberg, 2011). Additional 3D graphics were prepared with the help of Landmarks software. For statistical analyses which are not incorporated into MorphoJ software, Statistica 9 was used. Assumptions for statistical tests were tested by the ShapiroWilk's (normality) and Levene's tests (homogeneity of covariance matrices). For all tests, statistical significance was demarcated at the $5 \%$ level.

\section{RESULTS}

Prior to analysis processing the repeatability of data 
acquisition was tested upon a subsample of 25 bones, selected randomly from fused bones only. The measurement error was computed by the Procrustes ANOVA of shape variables design (Klingenberg \& McIntyre, 1998) and by two-way ANOVA for size variable (centroid size) with individuals and number of digitizing session as factors. The individual amount of variation exceeded the digitalization error by substantial amount (data not shown) suggesting than for this study, digitalization error is not a concern.

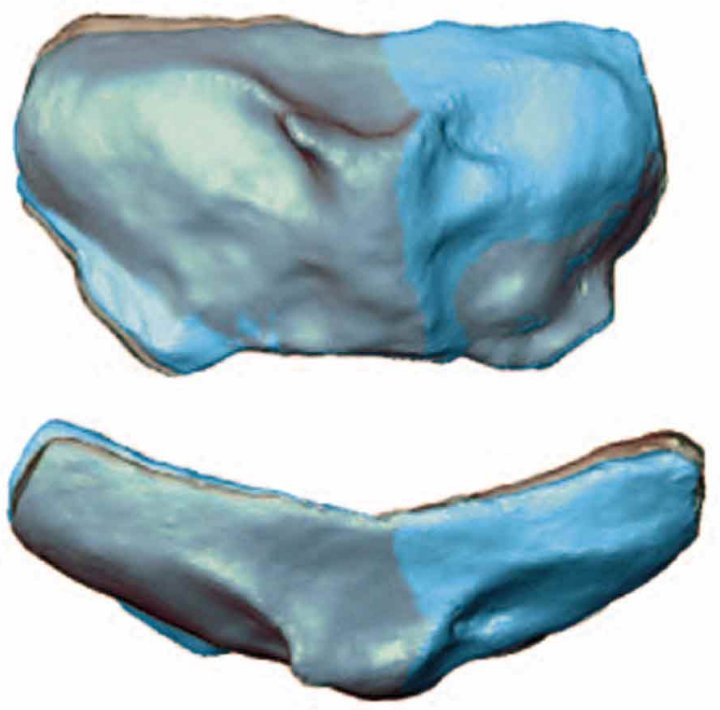

Fig.e 2. Superimposition of ideally symmetrical hyoid bone (orange) and hyoid of mean asymmetry (blue).
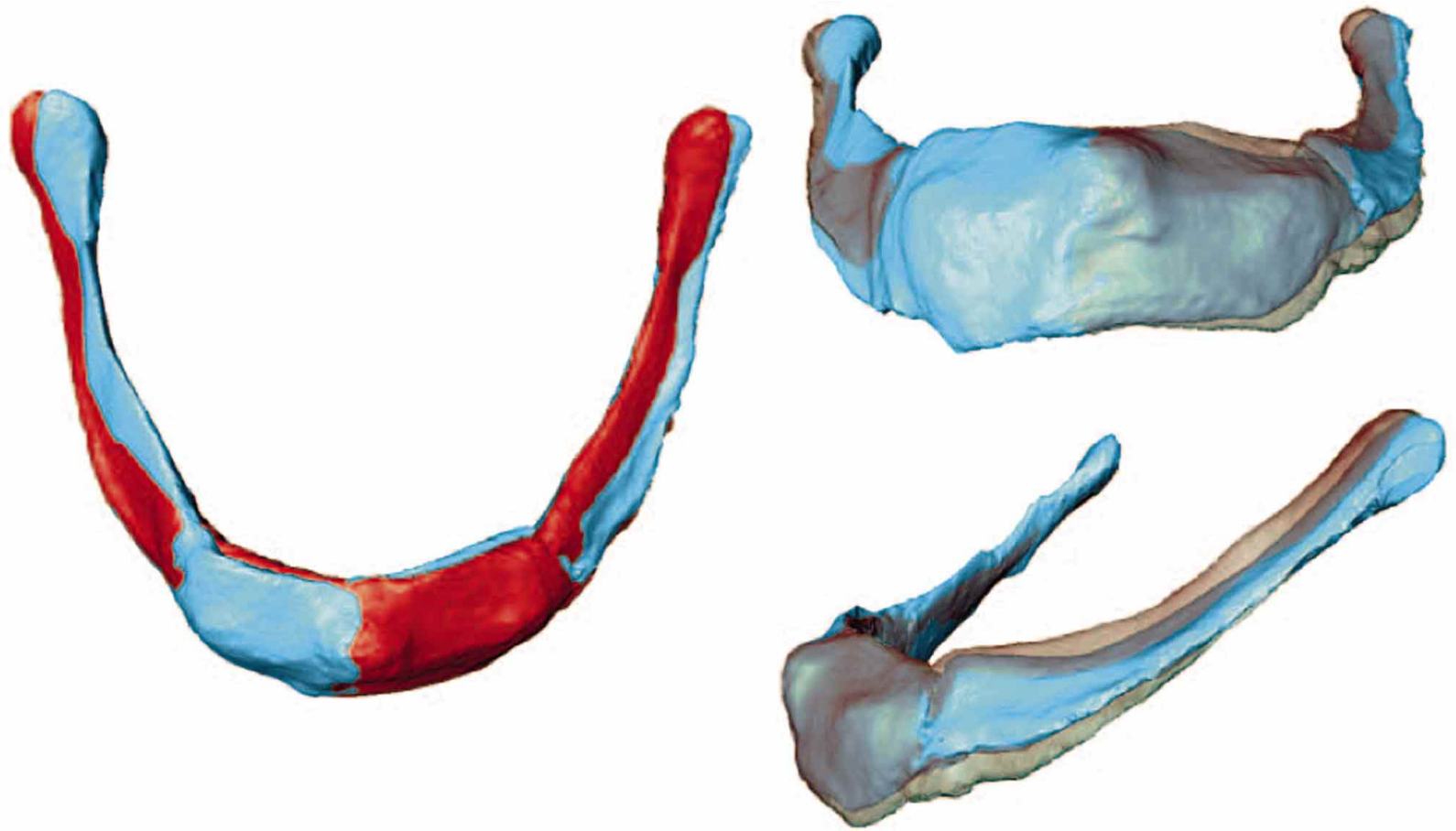

Fig. 3. Superimposition of ideally symmetrical hyoid body (orange) and body of mean asymmetry (blue) and right-left asymmetrical greater horn (blue) and average right greater horn (orange). 
Matching a/symmetry, greater horns, fused and nonfused bones $(\mathbf{n}=\mathbf{2 1 1})$. ANOVA showed statistically significant differences for directional asymmetry in size of greater horns if fused and non-fused bones are pooled (Table III). Right body side possesses larger greater horns than the left body side. Similarly, statistically significant individual differences in size were revealed (paired t-test, $\mathrm{t}=3.818$, $\mathrm{p}$ value $=0.0002)$. Similarly to object a/symmetry no interactions with individual's sex ( $\mathrm{t}$-test, $\mathrm{t}=0.547$, $\mathrm{p}$ value $=0.585$ ), age categories (ANOVA, $\mathrm{p}$-value $=0.924$ ) or occurrence of fusion (Mann-Whitney U test, $\mathrm{U}=-0.628$, $\mathrm{p}$ value $=0.530$ ) were observed.

Matching a/symmetry, greater horns, non-fused bones $(\mathbf{n}=\mathbf{4 5})$. The analysis provided comparable results when narrowed to non-fused bones only, i.e., on average larger greater horns on the right body side and statistically significant differences for a pair-wise comparison. However, sex-related differences were revealed for signed right and left differences where female bones exhibit, on average, larger values. If absolute value of side differences (unsigned scores) were tested, no sex-related differences were observed. Similarly, non-parametric comparison among age categories did not show age-dependency (Kruskal-Wallis test, $\mathrm{H}=3.644$, $\mathrm{p}$-value $=0.303$ ).

\section{A/symmetry in shape}

Object a/symmetry. Procrustes ANOVA yielded statistically significant results for asymmetry in the overall shape as well as the body. Based on a subsample of fused bones, the mean asymmetry was manifested in unequal sloping of the greater horns. On average, the right greater horns tend to curved inwards and upwards whereas the left greater horns direct down and laterally (Figs. 2 and 4). There is also an unequal extent of vertical flattening in the right and left greater horns as the left side tend to flatten to a larger degree. At the same time, the right half of the inferior margin of the body extended anteriorly, caudally and laterally (Figs. 3 and 4). The same applies for the asymmetry analysis of the body configurations $(n=211)$. Furthermore, an uneven distance between superior and inferior points of the lateral margin was also a source of right-left asymmetry (Figs. 3 and 4).

No sex-related or age-dependent variation was observed within fluctuating asymmetry (FA) scores as the Mann-Whitney U test and ANOVA yielded statistically insignificant results (data not shown). Significantly higher FA scores were revealed for the greater horns than for the body configuration (paired t-test, $\mathrm{t}=12.75$, $\mathrm{p}$-value $<0.0001$ ).

Table II. Procrustes ANOVA results for two types of a/symmetry expressed in shape and size variables within fused bones and separate anatomical elements.

\begin{tabular}{llccccc}
\hline & Effect & SS & MS & df & F & P(param.) \\
\hline Shape & Individual & 1.689 & 0.000320 & 5280 & 3.08 & $<0.0001$ \\
Object symmetry & Side & 0.061 & 0.002047 & 30 & 19.71 & $<0.0001$ \\
Fused bones & Ind*Side & 0.514 & 0.000104 & 4950 & & \\
Shape & Individual & 2.989 & 0.00129 & 2310 & 2.39 & $<0.0001$ \\
Object symmetry & Side & 0.108 & 0.01204 & 9 & 22.19 & $<0.0001$ \\
Body & Ind*Side & 1.025 & 0.00054 & 1890 & & \\
Centroid size (greater horns) & Individual & 0.00425 & 0.000020 & 210 & 1.64 & 0.0002 \\
Matching symmetry & Side & 0.00018 & 0.000180 & 1 & 14.58 & 0.0002 \\
& Ind*Side & 0.00260 & 0.000012 & 210 & & \\
Shape (greater horns) & Individual & 3.659 & 0.00124 & 2940 & 2.08 & $<0.0001$ \\
Matching symmetry & Side & 0.338 & 0.02416 & 14 & 40.31 & $<0.0001$ \\
Centroid size (non-fused greater & Ind*Side & 1.762 & 0.00060 & 2940 & & \\
horns) & Individual & 0.00010 & 0.00001 & 21 & 1.22 & 0.3281 \\
Matching symmetry & Side & 0.00003 & 0.00003 & 1 & 7.07 & 0.0147 \\
Shape (non-fused greater homs) & Ind*Side & 0.00009 & 0.00001 & 21 & & \\
Matching symmetry & Individual & 0.25380 & 0.00086 & 294 & 1.94 & $<0.0001$ \\
& Side & 0.008281 & 0.00059 & 14 & 1.33 & 0.1903 \\
\hline
\end{tabular}


Matching a/symmetry, greater horns, fused and non-fused bones $(\mathbf{n}=\mathbf{2 1 1})$. Statistically significant results in shape were shown for the directional asymmetry (Table II). The mean difference between right and left side was demonstrated in the overall width and flattening. Neither sex-, age- nor fusion-related variations were observed within the FA scores, as Mann-Whitney U test and ANOVA yielded statistically non-significant results.

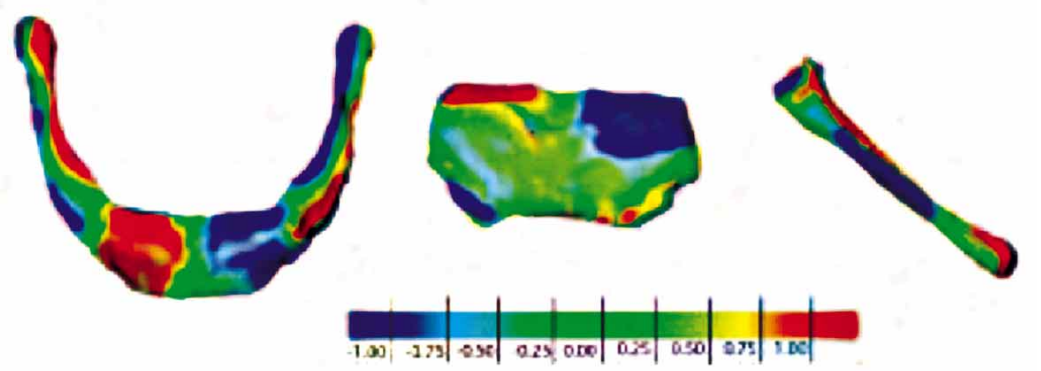

Fig. 4. Asymmetry in the hyoid bone, body and greater horn displayed in color scale.
Matching symmetry, greater horns, nonfused bones $(n=45)$. No statistically significant differences in directional asymmetry of non-fused greater horns were revealed (Table II).

Modularity. Of the 3 hypotheses of modularity, the lowest RV coefficients were achieved with modules partitioned with respect to different embryonic origin. The symmetrical component yielded consistently higher RV coefficients than the asymmetrical component. Minimal values of the RV coefficient were given by a partition wherein the body landmarks 5, 9 and 12 were set separately from landmarks of the inferior $(2$, $11,10,8,7,14)$ and superior margins $(4,6)$ (Table III).

Table III. Tested hypotheses of modularity. RV coefficient corresponds to the covariance among the tested modules (*Number of 10000 partitions considered).

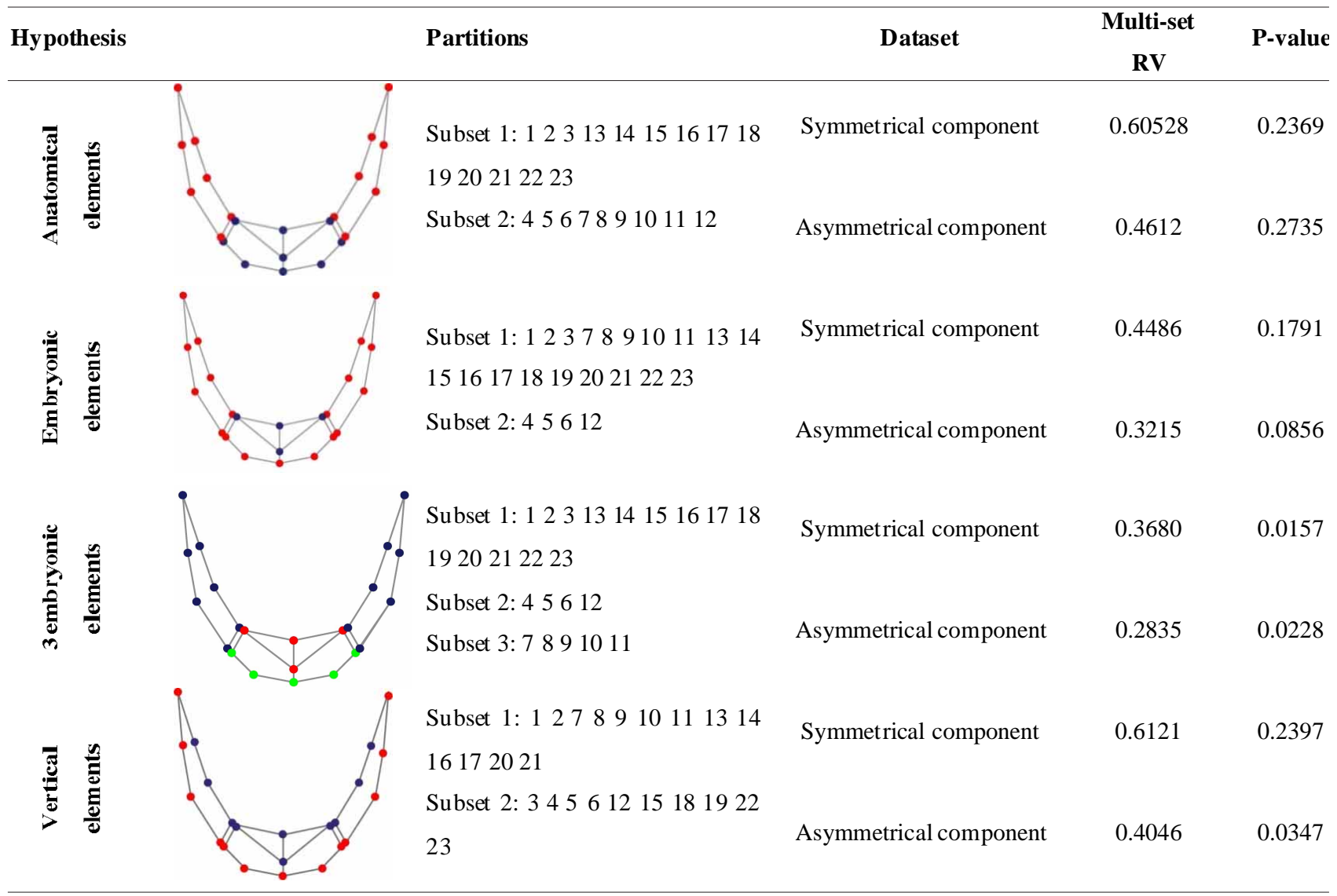




\section{DISCUSSION}

There is an undeniable presence of asymmetry in the studied sample of hyoid bones. The asymmetry is present in both the shape and size of the body and greater horns. Miller et al. noted that in hyoids, the asymmetry is concentrated in length rather than width measurements. In a paper by Shimizu et al. (2005), the asymmetry was concentrated in the breadth of the greater horns. In our sample, the asymmetry was manifested by the overall size, unequal length, sloping and curvature of greater horns, deviation of the midline body tubercle and an uneven course of the inferior body margin. On average, the hyoid body was more prominent anteriorly and extended down and outwards on its right side, while the right greater horn curved up and inwards. In biological studies, the average asymmetry is customarily ascribed to the directional asymmetry. The directional asymmetry can be a product of genotype as well as of lateralization in the muscle load or other functional demands (Klingenberg et al., 1998). In humans, the widely known example of directional asymmetry is laterality observable in the upper and lower limbs or the size and functions of the brain (Vallortigara et al., 1999). The most probable explanation for the observed average asymmetry in hyoid bones is that it results from a functional imbalance due to side preferences which are translated into unequal pull of paired muscles on bone. The hyoid is not in direct contact with any other bone of the skeleton. Without fixed or joint connection with other bony structures, the hyoid lacks compensatory mechanisms which are involved in retaining morphological equilibrium. These compensatory mechanisms were acknowledged, for instance, for bones of cranial vault (Ritchsmeier \& Deleon, 2009) or the facial skeleton (Figalová, 1969) where local defects are compensated by other parts of the structure. Therefore, any unilateral preference in muscle load would directly modulate external morphology.

The average asymmetry is more prominent in the greater horns than in the body. This is consistent with other studies where features located near the mid-sagittal plane tend to manifest a lesser degree of asymmetry. On the other hand, this seems to be irrelevant if a trait is strongly determined by unilateral functional demands rather than randomly distributed differences. For instance, Kolesnikov \& An (1999) mentioned a high degree of asymmetry in the nasal aperture and Hanson \& Owsley (1980) declared right-left asymmetry in the frontal sinuses. Both structures occupy areas in the median line but are misshaped by stronger pressure of the airflow in one of the airways.

In addition to directional asymmetry, hyoid bones exhibit a noticeable extent of fluctuating asymmetry.
Fluctuating asymmetry (FA) arises from diminutive random irregularities in otherwise bilateral development (Van Valen, 1962). At a population level, they can be observed as minor physical anomalies equally distributed on both body sides. Being a product of random acts, FA and its occurrence have been used as a measure of developmental instability (Klingenberg \& McIntyre). Since the right and left body side shares identical genetic coding, any within-individual right-left asymmetry can be ascribed to environmental impulses. Although this non-genetic basis of FA is not yet fully understood and is a question of ongoing disputes (Leamy \& Klingenberg 2005), the fluctuating asymmetry was previously implicated in malnutrition, living conditions, toxicity, gestational diabetes, obesity, smoking or alcoholism during pregnancy (Kieser et al., 1997; Møller \& Swaddle, 1997; Thornhill \& Møller, 1997; Singh \& Rosen, 2001). In the present study, none of the studied factors exhibited an association with the extent of FA (except for signed right-to-left scores of centroid size in non-fused greater horns which is likely due to sampling bias). This applies in particular to an individual's sex, which suggests that both sexes are susceptible to the same extent and that the hyoid bone may be resistant to the so-called "male-specific maternal immunoreactivity", which assumes that a mother's immune response to a male foetus increases developmental instability (Lalumière et al., 1999).

There is a lack of data on asymmetry in early stages of development of the hyoid bone. Therefore, for now we can only speculate at what point in an individual's life certain types of asymmetry occur. For the facial skeleton, for instance, the asymmetry reportedly exists from the early stages in development to elderly ages (Rossi et al., 2003). Being a product of developmental events, FA in the hyoid bone should be identically present at birth and then throughout life. For some of the somatic measurements, the extent of FA is somehow prone to diminish gradually by postnatal events (Wilson \& Manning, 1996). If valid in the case of the hyoid bone, we would have observed a decrease in Procrustes fluctuation scores with advancing age. No such age-dependent decrease was, however, yielded. By contrast, the directional asymmetry, being driven by biomechanical demands, would be expected to increase with age increment or at least to depend on biological sex. Our results failed, again, to confirm that the asymmetry changes with advancing age or is more apparent in male bones.

The comparison between the body and greater horns 
revealed that the greater horns are more inclined towards local disturbances. FA can be used as a tool to study patterns and amount of integration among anatomical parts in developmental processes as an uneven extent of FA among various compartments of the same anatomical structure is generally taken as an evidence for weaker within compartment interactions which are then more susceptible towards effects of environmental factors (Klingenberg et al., 2002). Given the independent lateral positions of the greater horns in contrast to the centrally placed singlestructured body it is certainly the case for the human hyoid bone.

We have shown that methods of geometric morphometric are very effective in exploring hypotheses about independency of skeletal units. The mammalian skull is highly integrated and evolutionary conserved system (Porto et al., 2009). Still, for the human craniofacial complex it has been noted that although the intra-module integration is very high the integration among traditional anatomical regions such as basicranium, facial skeleton and cranial vault are loosened in comparison to other species (Richtsmeier \& Deleon).

In regards to our results, there is a clear division of the hyoid bone into subdivisions, the first is the one that respects obvious anatomical units and the second, which was revealed as predominant, is the developmental compartmentalization due to a different embryonic origin. Even stronger integration was, however, revealed for the superior and inferior halves of the bone. Hyoid morphology is mostly determined by the biomechanical load of the suprahyoid and infrahyoid muscles. Hence, the morphology of the body co-varied with that of greater horns and so did the superior and inferior margins.

The symmetrical components yielded stronger covariance coefficients in anatomical subdivision as well as across the remaining tested hypotheses. The weaker integration of asymmetry is considered a widespread phenomenon as it was previously reported for other animal species, such as dogs, rodents or insects (Drake \& Klingenberg, 2010; Klingenberg, 2009).

It has been previously held that the hyoid bone originates from two embryonic structures. While the superior part of the body together with the lesser horns originate from the second pharyngeal arch, the greater horn and inferior part of the body originate from the third pharyngeal arch. Our results showed that partitions according to this dichotomy revealed a lower covariance than when anatomical or topographic elements were incorporated. This indicates that the superior and inferior parts of the body are distinct units and developmental integrations in the bone occur primarily within these units and not between them. Surprisingly, we were provided with an alternative scenario of three independent compartments which yielded the weakest theoretical dependency for all of the tested partitioning. According to the results, there appears to be a loosening of the otherwise homogenous interactions between the lateral parts and midline portion of the body. Due to a lack of appropriate landmarks on a structure, the exact location of the marginline delimiting these modules remained unresolved.

Recently, the concept of dual origin involving the second (hyoid) and third pharyngeal arches in the human hyoid has been questioned. Rodríguez-Vázquez et al. (2011) demonstrated on embryos that the hyoid body originates from a mesenchymal condensation that is separated from the second and third arch at an early stage of development and subsequently acts as an independent unit. By this, the hyoid bone would originate from the second and third pharyngeal arches for the lesser and greater horns respectively and mesenchymal cells separated most likely from the hypobrachial eminence "located at the base of the third pharyngeal arch" (Rodríguez-Vázquez et al.).

It is unclear whether this could translate to our results or is simply an unforeseen artifact of the analysis. Certainly further studies are required. For example, patterns of fluctuating asymmetry within each module and their correlation could shed light on the extent of developmental interactions. Two modules will be correlated if there are developmental interactions between them that can transmit the effect to both structures, for example, the presence of a common precursor. If, however, they develop separately, without any interactions, actions of a stressor cannot be transmitted (Leamy \& Klingenberg). Unfortunately, there are a limited number of modules that are suitable for this particular investigation. First of all, one would have to deal with the technical difficulty concerning the manner by which the morphology is described and modules are delimited. Due to ossification and ongoing bone remodeling in adult skeletal structures, original embryonic modules are almost impossible to recognize by an external examination. For hyoid bones, specifically, it would most likely require a set of additional reliable landmarks which on a structure like the hyoid bone are uneasy to define. A collective study involving other skeletal structures of identical embryonic origin, such as the styloid process, stapes or thyroid cartilage could be of help. Furthermore, a study which would incorporate the lesser horns into the analysis could further clarify these interactions. 


\section{CONCLUSION}

This study represents a contribution to morphological studies of the human hyoid bone. In terms of covariance, the studied anatomical elements, e.g. body and greater horns, form relatively strongly integrated units. It was demonstrated that modern analytical tools can provide an insight to patterns of this integration. The modularity and asymmetry analyses demonstrated that, by taking account only adult morphology, they have the capacity to clarify longer lengths of various developmental trajectories which may go back to early stages of development.

URBANOVÁ, P.; HEJNA, P.; ZÁTOPKOVÁ, L. \& SAFR, M. Asimetría y modularidad del hueso hioides. Int. J. Morphol., 32(1):251$260,2014$.

RESUMEN: La variación morfológica es el resultado de la interacción entre múltiples factores. Para huesos hioides, las diferencias de forma y tamaño han sido poco mencionadas en la literatura y se limitan a estudios del dimorfismo sexual o distribución etaria. Hasta donde sabemos, el hueso hioides humano, a diferencia de otros huesos craneales, no ha sido utilizado para hacer frente a interrogantes del desarrollo en términos de asimetría o de la modularidad. Utilizamos métodos basados en hitos de la morfometría geométrica y en el enfoque estadístico multivariado para estudiar la morfología del hueso hioides humano, representado por el cuerpo del hioides y astas mayores, en una muestra de 211 huesos fusionados y no fusionados. En un análisis de la variación de la muestra, se demostró que el hueso hioides es por naturaleza un hueso asimétrico, que exhibe tipos de asimetría tanto direccionales y fluctuantes, compuesto de elementos anatómicos bien integrados para los cuales, la carga biomecánica de músculos vinculados es el factor más determinante de la variación. Sin embargo, la covarianza y la evidencia de la cantidad desigual de asimetría fluctuante entre módulos sugiereun cierto grado de independencia durante las primeras etapas de desarrollo.

PALABRAS CLAVE: Hueso hioides; Asimetría; Modularidad; Morfometría geométrica.

\section{REFERENCES}

Drake, A. G. \& Klingenberg, C. P. Large-scale diversification of skull shape in domestic dogs: disparity and modularity. Am. Nat., 175(3):289-301, 2010.

Figalová, P. Asymmetry of the face. Anthropologie, 7(1):31-4, 1969.

Gupta, A.; Kohli, A.; Aggarwak, N. K. \& Banerjee, K. K. Study of age of fusion of hyoid bone. Legal Med. (Tokyo), 10(5):253-6, 2008 .

Hanson, C. L. \& Owsley, D. W. Frontal sinus size in Eskimo populations. Am. J. Phys. Anthropol., 53(2):251-5, 1980.

Kieser, J. A.; Groeneveld, H. T. \& Da Silva, P. C. Dental asymmetry, maternal obesity, and smoking. Am. J. Phys. Anthropol., 102(1):133-9, 1997.

Kindschuh, S. C.; Dupras, T. L. \& Cowgill, L. W. Determination of sex from the hyoid bone. Am. J. Phys. Anthropol., 143(2):279-84, 2010.

Kindschuh, S. C.; Dupras, T. L. \& Cowgill, L. W. Exploring ancestral variation of the hyoid. J. Forensic Sci., 57(1):41-6, 2012.

Klingenberg, C. P. Morphological integration and developmental modularity. Annu. Rev. Ecol. Evol. Syst., 39:115-32, 2008.
Klingenberg, C. P. Morphometric integration and modularity in configurations of landmarks: Tools for evaluating a-priori hypotheses. Evol. Dev., 11(4):405-21, 2009.

Klingenberg, C. P. MorphoJ: an integrated software package for geometric morphometrics. Mol. Ecol. Resour., 11(2):353-7, 2011.

Klingenberg, C. P.; Barluenga, M. \& Meyer, A. Shape analysis of symmetric structures: quantifying variation among individuals and asymmetry. Evolution, 56(10):1909-20, 2002.

Klingenberg, C. P. \& McIntyre, G. S. Geometric morphometrics of developmental instability: analyzing patterns of fluctuating asymmetry with Procrustes methods. Evolution, 52(5):136375, 1998.

Klingenberg, C. P.; McIntyre, G. S. \& Zaklan, S. D. Left-right asymmetry of the fly wings and the evolution of body axes. Proc. Biol. Sci., 265(1402):1255-9, 1998.

Kolesnikov, L. L. \& An, S. V. Asymmetry of the facial skeleton in Negroid skulls. Stomatologiia (Mosk), 78(4):39-41, 1999.

Lalumière, M. L.; Harris, G. T. \& Marnie, E. R. Birth order and fluctuating asymmetry: a first look. Proc. Biol. Sci., 266(1436):2351-4, 1999. 
Leamy, L. J. \& Klingenberg, C. P. The genetics and evolution of fluctuating asymmetry. Annu. Rev. Ecol. Evol. Syst., 36:1-21, 2005 .

Miller, K. W.; Walker, P. L. \& O'Halloran, R. L. Age and sex-related variation in hyoid bone morphology. J. Forensic Sci., 43(6):1138-43, 1998.

Møller, A. P. \& Swaddle, J. P. Asymmetry, developmental stability, and evolution. Oxford, Oxford University Press, 1997. p.291.

Palmer, A. R. \& Strocker, C. Fluctuating asymmetry: measurement, analysis, patterns. Annu. Rev. Ecol. Evol. Syst., 17:391-421, 1986.

Pollanen, M. \& Ubelaker, D. H. Forensic significance of the polymorphism of hyoid bone shape. J. Forensic Sci., 42(5):8902, 1997.

Porto, A.; de Oliveira, F. B.; Shirai, L. T.; De Conto, V. \& Marroig, G. The evolution of modularity in the mammalian skull I: Morphological integration patterns and magnitudes. Evol. Biol., 36:118-35, 2009.

Richtsmeier, J. T. \& Deleon, V. B. Morphological integration of the skull in craniofacial anomalies. Orthod. Craniofac. Res., 12(3):149-58, 2009

Rodríguez-Vásquez, J. F.; Kim, J. H.; Verdugo-López, S.; Murakami, G.; Cho, K. H.; Asakawa, S. \& Abe, S. Human fetal hyoid bone origin revisited. J. Anat., 219(2):143-9, 2011.

Rossi, M.; Ribeiro, E. \& Smith, R. Craniofacial asymmetry in development: An anatomical study. Angle Orthod., 73(4):3815, 2003.

Scheuer, L. \& Black, S. Developmental juvenile osteology. London, Elsevier Ltd., 2000.

Shimizu, Y.; Kanetaka, H.; Kim, Y. H.; Okayama, K.; Kano, M. \& Kikuchi, M. Age-related morphological changes in the human hyoid bone. Cells Tissues Organs, 180(3):185-92, 2005.

Singh, D. \& Rosen, V. C. Effect of maternal body morphology, morning sickness, gestational diabetes and hypertension on fluctuating asymmetry in young women. Evol. Hum. Behav., 22(6):373-84, 2001.

Thornhill, R. \& Møller, A. P. Developmental stability, disease and medicine. Biol. Rev. Camb. Philos. Soc., 72(4):497-548, 1997.

Urbanová, P.; Hejna, P.; Zátopková, L. \& S`afr, M. What is the appropriate approach in sex determination of hyoid bones? $J$. Forensic Leg. Med., 20(8):996-1003, 2013a.

Urbanová, P.; Hejna, P.; Zátopková, L. \& S`afr, M. The morphology of human hyoid bone in relation to sex, age and body proportions. Homo, 64(3):190-204, 2013 b.
Vallortigara, G.; Rogers, L. J. \& Bisazza, A. Possible evolutionary origins of cognitive brain lateralization. Brain Res. Brain Rev., 30(2):164-75 1999.

Van Valen, L. A study of fluctuating asymmetry. Evolution 16(2):125-42, 1962.

Wilson, J. M. \& Manning, J. Fluctuating asymmetry and age in children: evolutionary implications for the control of developmental stability. J. Hum. Evol., 30(6):529-37, 1996.

Correspondence to:

Department of Anthropology

Faculty of Science

Masaryk University

Kotlárská 2, 61137 Brno

CZECH REPUBLIC

Email: urbanova@sci.muni.cz

Received: 19-09-2013

Accepted: 19-11-2013 